\title{
Oxide-cladding aluminum nitride photonic crystal slab: Design and investigation of material dispersion and fabrication induced disorder
}

Cite as: J. Appl. Phys. 119, 023107 (2016); https://doi.org/10.1063/1.4939773

Submitted: 16 October 2015 . Accepted: 29 December 2015 . Published Online: 13 January 2016

E. G. Melo (D), D. O. Carvalho (D), A. S. Ferlauto, M. A. Alvarado, M. N. P. Carreño, and M. I. Alayo (D)
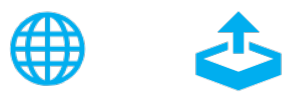

\section{ARTICLES YOU MAY BE INTERESTED IN}

Aluminum nitride nanophotonic circuits operating at ultraviolet wavelengths Applied Physics Letters 104, 091108 (2014); https://doi.org/10.1063/1.4867529

Near-infrared gallium nitride two-dimensional photonic crystal platform on silicon Applied Physics Letters 105, 011104 (2014); https://doi.org/10.1063/1.4887065

Gallium nitride L3 photonic crystal cavities with an average quality factor of 16900 in the near infrared

Applied Physics Letters 105, 231119 (2014); https://doi.org/10.1063/1.4903861 


\title{
Oxide-cladding aluminum nitride photonic crystal slab: Design and investigation of material dispersion and fabrication induced disorder
}

\author{
E. G. Melo, ${ }^{1, a)}$ D. O. Carvalho, ${ }^{2}$ A. S. Ferlauto, ${ }^{3}$ M. A. Alvarado, ${ }^{1}$ M. N. P. Carreño, ${ }^{1}$ \\ and M. I. Alayo ${ }^{1}$ \\ ${ }^{1}$ Electronic Systems Engineering Department, University of São Paulo, CEP 05508-010 São Paulo, SP, Brazil \\ ${ }^{2}$ UNESP - São Paulo State University, CEP 13874-149 São João da Boa Vista, SP, Brazil \\ ${ }^{3}$ Department of Physics, Federal University of Minas Gerais, CEP 31270-901 Belo Horizonte, MG, Brazil
}

(Received 16 October 2015; accepted 29 December 2015; published online 13 January 2016)

\begin{abstract}
Photonic crystal slabs with a lower-index material surrounding the core layer are an attractive choice to circumvent the drawbacks in the fabrication of membranes suspended in air. In this work we propose a photonic crystal $(\mathrm{PhC})$ slab structure composed of a triangular pattern of air holes in a multilayer thin film of aluminum nitride embedded in silicon dioxide layers designed for operating around $450 \mathrm{~nm}$ wavelengths. We show the design of an ideal structure and analyze the effects of material dispersion based on a first-order correction perturbation theory approach using dielectric functions obtained by experimental measurements of the thin film materials. Numerical methods were used to investigate the effects of fabrication induced disorder of typical nanofabrication processes on the bandgap size and spectral response of the proposed device. Deviation in holes radii and positions were introduced in the proposed $\mathrm{PhC}$ slab model with a Gaussian distribution profile. Impacts of slope in holes sidewalls that might result from the dry etching of AlN were also evaluated. The results show that for operation at the midgap frequency, slope in holes sidewalls is more critical than displacements in holes sizes and positions. (C) 2016 AIP Publishing LLC.
\end{abstract}

[http://dx.doi.org/10.1063/1.4939773]

\section{INTRODUCTION}

Photonic crystal $(\mathrm{PhC})$ slabs combine both the bandgap effect and the vertical index guiding confinement to control and manipulate electromagnetic radiation. ${ }^{1,2}$ Beyond the traditional materials with high refractive index (i.e., $\mathrm{Si}$ and $\mathrm{GaAs}$ ) working in the telecommunication window, widebandgap materials have allowed the researches on PhC slabs to explore the wavelengths of visible and ultraviolet radiation; thus, a novel set of potential applications, such as efficient light extraction in blue and ultraviolet optoelectronic devices, ${ }^{3}$ near-ultraviolet biosensors, ${ }^{4}$ enhanced fluorescence emission from quantum dots, ${ }^{5}$ and others, has emerged.

Aluminum nitride (AlN) has suitable physical properties for nanophotonics. Its large electronic bandgap $(6.20 \mathrm{eV})$ at room temperature makes it transparent to electromagnetic radiation ranging from infrared to deep ultraviolet wavelengths. AlN exhibits appreciable second and third-order nonlinear susceptibilities that are very attractive for applications such as electro-optic modulation, second harmonic generation, ${ }^{6}$ and frequency comb generation. ${ }^{7}$ Furthermore, AlN is a promising candidate for robust integration with high power photonics and microelectronics as it is more tolerant to temperature fluctuations than silicon ${ }^{8}$ due to its small thermo-optic ${ }^{9}$ and expansion ${ }^{10}$ coefficients, and its large thermal conductivity. ${ }^{11}$

Recent advances have been reported in the fabrication of high-Q nanocavities embedded in air-bridge AlN PhC slabs. ${ }^{12-14}$ Nevertheless, from a practical point of view,

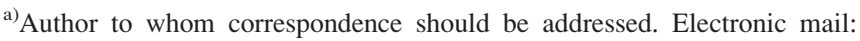
emerdemelo@usp.br
}

air-bridge structures can have a few shortcomings: (i) the membrane structure is both fragile and unstable, and it needs supporting nanotethers and cannot cover a large area; (ii) its processing requires the removal of a silicon dioxide $\left(\mathrm{SiO}_{2}\right)$ or silicon layer underneath the $\mathrm{PhC}$ by isotropic etching, which makes it difficult to integrate these PhC devices with other silicon microelectronics or photonics devices on the same chip; and (iii) optical transitions between dielectric channel waveguides with and without a supporting substrate might give undesired reflections and mode conversions.

To circumvent these drawbacks, a more practical system can be made by adding a lower-index cladding on both sides of the slab so that the mirror symmetry, which is crucial for the existence of larger photonic bandgaps ${ }^{15}$ (PBG), is preserved. Nevertheless, intrinsic loss can be increased as a consequence of the reduction in index contrast, ${ }^{2}$ and there are other issues that may also contribute to degrade device performance. One such issue is the presence of fabrication induced disorder as fluctuations in holes size and position or sloped sidewalls and surface roughness that contribute to increase the amount of extrinsic optical scattering loss. ${ }^{16}$

In face of all the fabrication challenges in the patterning of group III-nitride materials it is necessary to carefully analyze their benefits. Thus, considering the great potential of AlN for the fabrication of integrated photonics and microelectronics devices as well as a more amenable fabrication process, we propose an oxide-cladding AlN PhC slab structure. Its design was conducted in order to achieve a maximum PBG, so that it can efficiently support line and point defect states with low intrinsic losses below the light line. We choose short operation wavelengths, around $450 \mathrm{~nm}$, for 
this study so as to enable the analysis of the device performance under critical feature sizes, such as those needed in the aforementioned references. ${ }^{3-5,12-14}$ Initially, we present the characteristics of an ideal structure and compare the results with its counterpart air-bridge type, and so, we analyze the impacts of material dispersion and fabrication induced disorder on the PBG size and spectral response of the proposed $\mathrm{PhC}$.

The design of the proposed device was preceded by an experimental investigation of the dielectric functions of sputtered deposited $\mathrm{AlN}$ and $\mathrm{SiO}_{2}$ thin films by variable angle of incidence spectroscopic ellipsometry (VASE) measurements. Thus, the influence of material dispersion in the $\mathrm{PhC}$ band diagram was determined by a first-order perturbation theory approach. Fabrication induced disorder effects were successfully addressed both analytically and numerically ${ }^{17-21}$ and so, we employed the finite-differences time-domain (FDTD) method to theoretically investigate the effects of disorder that can be introduced in nanofabrication processes, such as electron-beam lithography and dry etching techniques. Deviation in the holes radii and positions were introduced in the proposed PhC slab model following a Gaussian distribution profile, which matches very well with experimental verifications. ${ }^{21}$ Sloped sidewalls are also a common issue in dry etching of $\mathrm{AlN},{ }^{8,13}$ and their effects are also evaluated in this work.

\section{MATERIALS AND METHODS}

The proposed $\mathrm{PhC}$ slab structure studied in this work is composed of a triangular pattern of air holes perforated in a multilayer thin film made of an AIN core surrounded by two $\mathrm{SiO}_{2}$ cladding layers. Figure 1(a) shows the unit cell of the ideal $\mathrm{PhC}$ slab with lattice constant $a$, air hole radius $r$, AlN layer thickness $h$, oxide-cladding layer thickness $h_{c}$, and the outer media, i.e., a thick thermally grown $\mathrm{SiO}_{2}$ insulation layer or a upper air layer, with thickness $h_{o}=6 a$. In comparison with a bulk cladding, etching together the claddings and core materials have some advantages such as ease of fabrication and enhanced confinement of guided modes below the light cone. ${ }^{2}$ In comparison with other group III-V materials, another advantage of using AlN as a core layer is the possibility of fabricating the multilayer thin film by a sequential deposition process with sputtering techniques.

The $\mathrm{PhC}$ slab design was preceded by experimental measurements of the $\mathrm{AlN}$ and $\mathrm{SiO}_{2}$ dielectric functions through VASE measurements. For this purpose, thin films of AlN and $\mathrm{SiO}_{2}$ were deposited at room temperature by rf magnetron sputtering on separate Si (100) wafers. Good quality highly oriented wurtzite AIN films were obtained following deposition conditions already reported in a previous work. ${ }^{22}$

The VASE measurements were performed in a rotating compensator M2000 Woollam having spectral range from $246 \mathrm{~nm}$ to $1689 \mathrm{~nm}$ and using 4 incidence angles $\left(55^{\circ}\right.$ to $70^{\circ}$ in $5^{\circ}$ steps). The optical response of the films in the transparent region were described by a 2-term Sellmeier formula in which the dielectric function $\epsilon=\epsilon_{1}+i \epsilon_{2}$ is given by Eq. (1), where $A$ and $\lambda_{0}$ are the amplitude and wavelength position of a undamped Lorentz oscillator in the UV region, respectively, $A_{I R}$ is the amplitude of a undamped Lorentz oscillator with zero resonance energy, and $\epsilon_{\infty}$ is a contribution due to absorption at much higher photon energies.

$$
\begin{aligned}
& \epsilon_{1}=n^{2}=\epsilon_{\infty}+A \frac{\lambda^{2}}{\lambda^{2}-\lambda_{0}^{2}}-A_{I R} \lambda^{2}, \\
& \epsilon_{2}=0 .
\end{aligned}
$$

In order to analyze the AlN film spectra, an anisotropic uniaxial refractive index was employed to account for the preferential crystallographic orientation of the film given by the epitaxial like growth. ${ }^{23}$ The uniaxial refractive index has two components, the extraordinary $\left(n_{e}\right)$, which is perpendicular to the film surface, and the ordinary $\left(n_{o}\right)$, which represents the response parallel to the film plane. The adjustment of the VASE was restricted to photon energies smaller than $3.4 \mathrm{eV}(\lambda=365 \mathrm{~nm})$ because above this value AlN has a small but significant absorption ${ }^{24}$ that impedes a straightforward analysis using anisotropic optical functions. The VASE spectra for the $\mathrm{SiO}_{2}$ film were adjusted within the entire measured range. Table I presents the results for the adjusted

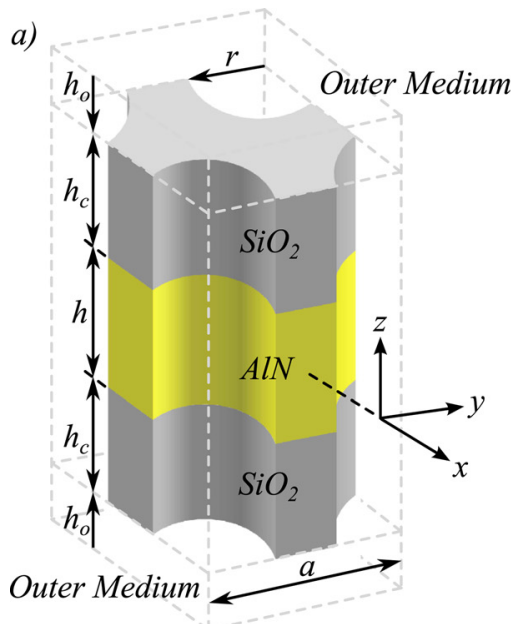

b)

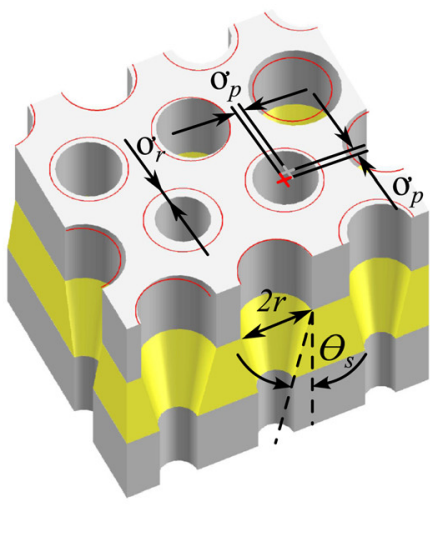

c)

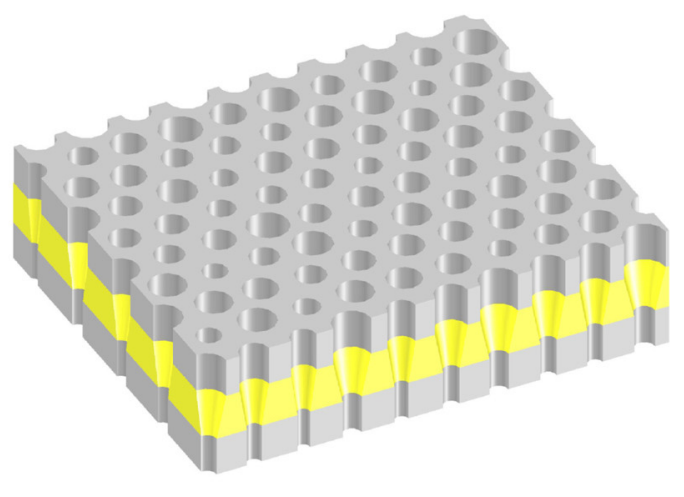

FIG. 1. (a) Diagram of the ideal PhC slab unit cell with lattice constant $a$, hole radius $r$, AlN layer thickness $h$, oxide-cladding layer thickness $h_{c}$, and outer media $h_{o}=6 a$. (b) Detail of a disordered PhC structure defined by the standard deviations in holes radius $\sigma_{r}$ and positions $\sigma_{p}$, and by the slope angle $\theta_{s}$. (c) Example of a PhC structure in which all the three perturbations analyzed in this work were introduced. 
TABLE I. Coefficients of the 2-term Sellmeier formula used to fit the dielectric functions of $\mathrm{SiO}_{2}$ and $\mathrm{AlN}$ thin films.

\begin{tabular}{lcccc}
\hline \hline Material & $\epsilon_{\infty}$ & $A$ & $\lambda_{0}(\mathrm{~nm})$ & $A_{I R}$ \\
\hline $\mathrm{SiO}_{2}$ & $1.62(0.03)$ & $0.521(0.031)$ & $121(3)$ & $\left.\begin{array}{c}1.23 \times 10^{-2} \\
\left(0.04 \times 10^{-2}\right.\end{array}\right)$ \\
$\mathrm{AlN}\left(n_{o}\right)$ & $2.48(0.08)$ & $1.80(0.08)$ & $175(2)$ & $\left.\begin{array}{c}2.47 \times 10^{-2} \\
\left(0.11 \times 10^{-2}\right.\end{array}\right)$ \\
$\mathrm{AlN}\left(n_{e}\right)$ & $4.00(0.05)$ & $0.435(0.039)$ & $279(5)$ & 0 \\
\hline \hline
\end{tabular}

Sellmeier parameters. The obtained spectra for the refractive index of $\mathrm{SiO}_{2}$ and $\mathrm{AlN}$ are very similar to those determined in previous works. ${ }^{23,25}$

The parameters of the unit cell of the ideal PhC slab structure exhibited in Fig. 1(a) were normalized by the lattice constant $a$. Considering the normalized cladding thickness $h_{c} / a$ extending infinitely into the $z$ direction, the normalized radius $r / a$ and core AlN layer thickness $h / a$ were defined in order to maximize the PBG in the $\Gamma-K$ direction for inplane electric field propagation (TE-like polarization). The range of $r / a$ values was defined based on the PBG map of the two-dimensional $\mathrm{PhC}$ structure ${ }^{26}$ and so, the gap-midgap ratios $\left(\Delta \omega / \omega_{m}\right)$ were calculated from the band diagrams computed using a three-dimensional plane wave expansion (PWE) implementation. ${ }^{27}$ The anisotropy of the AlN was considered in all the calculations.

The oxide-cladding normalized thickness $h_{c} / a$ was defined as a minimum one necessary to isolate the evanescent fields from the outer media. If a lossy material is introduced in the outer medium, the absorption loss caused by it in this region follows the relationship $\alpha_{A B S} \propto f_{m},{ }^{28}$ in which the modal field fraction $f_{m}$ is defined in Eq. (2) as the ratio between the electric field energy in the volume $V_{A B S}$, related to an absorptive material that might lie in the outer media, and the total electric field energy in the unit cell volume $V$. The minimization of $f_{m}$ ensures that extended states will not be perturbed, independently of the properties of the materials below and above the oxide-cladding layers, and thus the symmetry of the modes in relation to the plane $x y$ bisecting the core will be preserved.

$$
f_{m}=\frac{\int_{V_{A B S}} d^{3} \boldsymbol{r} \varepsilon(\boldsymbol{r})|\boldsymbol{E}(\boldsymbol{r})|^{2}}{\int_{V} d^{3} \boldsymbol{r} \varepsilon(\boldsymbol{r})|\boldsymbol{E}(\boldsymbol{r})|^{2}} .
$$

The band diagrams corrected for material dispersion were given by Eq. (3), which shows the well-known first order correction perturbation theory approach employed to obtain the frequencies $\omega^{(1)}$ from the unperturbed values $\omega^{(0)}$ corrected by the small perturbation $\Delta \varepsilon(\boldsymbol{r})$ in the position dependent dielectric function $\varepsilon(\boldsymbol{r}) .{ }^{29}$

$$
\omega^{(1)}=\omega^{(0)}-\frac{\omega^{(0)}}{2} \frac{\int d^{3} \boldsymbol{r} \Delta \varepsilon(\boldsymbol{r})|\boldsymbol{E}(\boldsymbol{r})|^{2}}{\int d^{3} \boldsymbol{r} \varepsilon(\boldsymbol{r})|\boldsymbol{E}(\boldsymbol{r})|^{2}} .
$$

Fabrication induced disorders were considered separately from those introduced in the lithography process (deviations in holes sizes and positions) and those originated in the dry etching step (holes with sloped sidewalls). Their influences on device performance, especially in gap-midgap ratio and midgap frequency, were observed by computing the transmission, reflection, and radiation spectra using 3DFDTD. ${ }^{30}$ The transmission and reflection spectra were split into straight and scattered ones. All the simulations were performed using a grid size of $a / 20$, and the three-dimensional oxide-cladding AlN PhC slab model was surrounded by an external air layer and a perfectly matched layer able to absorb all the incoming radiation.

Fan et al. ${ }^{21}$ demonstrate that disorder in $\mathrm{PhC}$ holes sizes and positions can be better represented by a Gaussian distribution profile instead of a continuous distribution. Thus, fluctuations in sizes and positions of the holes were introduced in our model through an automated computational routine that randomly changes the radius and/or positions of the $\mathrm{PhC}$ holes following a Gaussian distribution profile. The parameters $\sigma_{r}$ and $\sigma_{p}$ shown in Fig. 1(b) are, respectively, the standard deviations of the fluctuations in holes radius and shifts in holes positions with respect to their $x$ and $y$ coordinates.

In order to analyze the impacts of slope in holes sidewalls we calculate the spectra resulting from a variable slope angle $\left(\theta_{s}\right)$ attributed to the holes sidewalls in the AlN core layer. In $\mathrm{SiO}_{2}$ layers, the holes have a constant radius, but at the upper layer it is the nominal $r$ value, whereas at the bottom layer it is defined by the angle $\theta_{s}$. Fig. 1(c) shows an example of a $\mathrm{PhC}$ structure in which all the three perturbations analyzed in this work were introduced. All the analyses were realized considering negligible roughness in the holes sidewalls.

\section{RESULTS AND DISCUSSION}

The gap-midgap ratio of the maximum PBG in the $\Gamma-K$ direction, as a function of $h / a$ and $r / a$, is presented in Fig. 2. The optimum point occurs at $h / a=0.90$ and $r / a=0.35$ and corresponds to a partial PBG between the

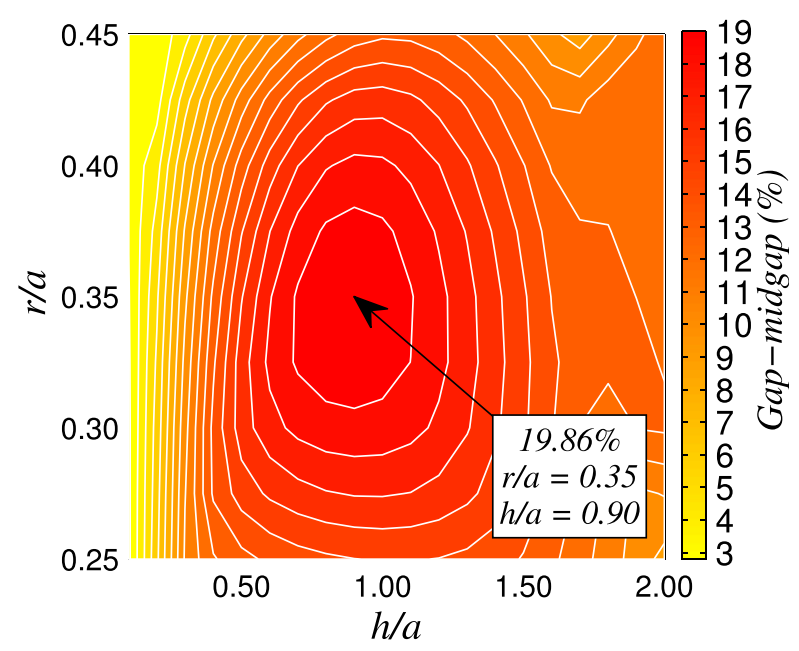

FIG. 2. PhC slab gap-midgap ratio computed in the $\Gamma-K$ direction as a function of the normalized AlN layer thickness $h / a$ and normalized radius $r / a$. The oxide-cladding thickness was considered to extend infinitely in the $z$ direction. The maximum gap-midgap ratio is $19.86 \%$ and occurs for $h / a=0.90$ and $r / a=0.35$. 
first two lowest bands. The gap-midgap ratio of $19.86 \%$, still considering an infinite oxide-cladding layer, is the maximum in this situation. We can also observe a large $r / a$ and $h / a$ range inside the continuous contour corresponding to $19 \%$ gap-midgap ratio. This provides more amenable fabrication tolerances with respect to hole radius and AIN film thickness fluctuations.

Fig. 3 shows the $f_{m}$ as well as the gap-midgap ratio values for $h_{c} / a$ ranging from 0.1 to 2.0. All these curves exhibit an exponential profile with their maximum at $h_{c} / a=0.1$, which represents roughly the same structure as an air-bridge $\mathrm{PhC}$ slab. Near $h_{c} / a=1.5$ a steady state condition is achieved and the values approximate the ones in the previous infinite oxide-cladding layer structure. While the transition from $h_{c} / a=0.1$ to $h_{c} / a=2.0$ takes place, the evanescent fields of both modes become increasingly confined at the claddings, but meanwhile, the effective dielectric constant surrounding the $\mathrm{PhC}$ core layer become higher, so that it pushes the second band toward lower frequencies and shrinks the gap-midgap ratio. Thus, the best oxide-cladding layer thickness was established with the purpose of achieving a good compromise among the electromagnetic field insulation from the outer media, a higher gap-midgap ratio at the $\Gamma-K$ direction, and also a reduced multilayer thin film height in order to keep the aspect ratio as low as possible. Based on data of Fig. 3 we observe that $h_{c} / a=0.8$ is a good choice to encompass all these requirements.

The effect of AlN material dispersion in the $\mathrm{PhC}$ slab eigenstates was also analyzed. The shifts $\delta \omega=\omega^{(1)}-\omega^{(0)}$ in the unperturbed eigenvalues $\omega^{(0)}$, as a consequence of the experimentally obtained AIN dispersion functions, can be seen in Fig. 4, in which the continuous curves are the unperturbed bands and the square marks are the perturbed ones. In general, the values of $\delta \omega$ were negligible but it were a little more pronounced in the higher order bands. The gap-midgap ratio suffers a reduction of only $0.85 \%$ in the $\Gamma-K$ direction, a value very low to significantly impact the device performance. However, the shifts could be more intense if it

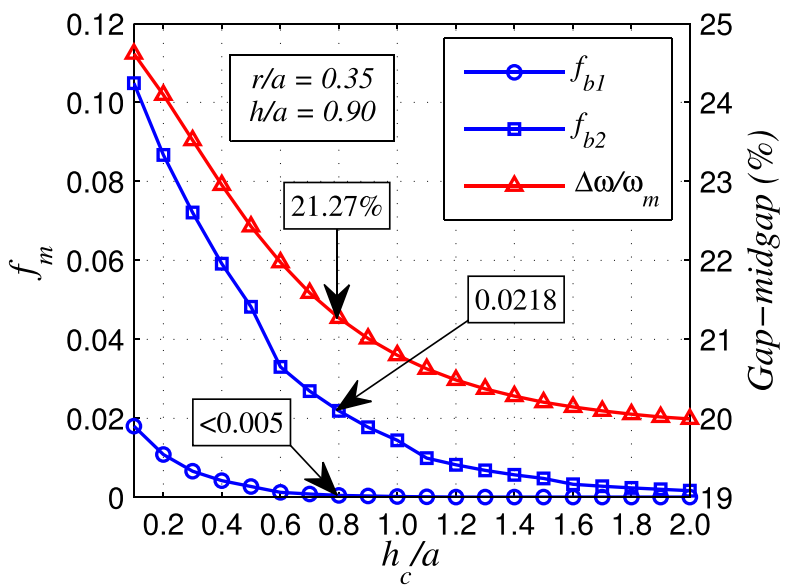

FIG. 3. Modal filling fraction $f_{m}$ and gap-midgap ratio in the $\Gamma-K$ direction as a function of the oxide-cladding normalized layer thickness $h_{c} / a$. The partial PBG opens between the bands 1 and 2. A good compromise among the electromagnetic field insulation, higher gap-midgap ratio, and a reduced multilayer stack height occurs at $h_{c} / a=0.80$.

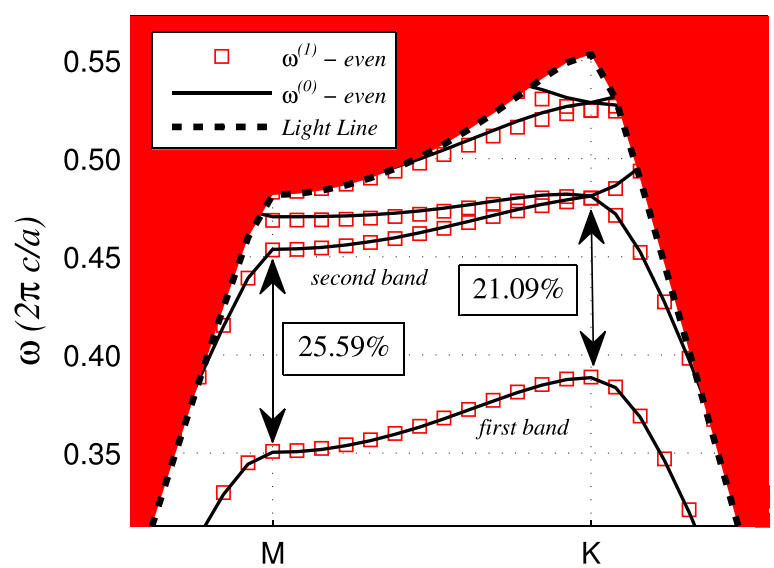

FIG. 4. Comparison of photonic bands of a dispersionless AlN oxidecladding PhC slab $\left(\omega^{(0)}\right)$ and a dispersive one $\left(\omega^{(1)}\right)$ around the directions $\Gamma-M$ and $\Gamma-K$. There are negligible shifts in the perturbed eigenvalues $\omega^{(1)}$ in relation to the unperturbed ones. The first order corrected gapmidgap ratios in the $\Gamma-K$ and $\Gamma-M$ directions are $21.09 \%$ and $25.59 \%$, respectively.

were not for the poor confinement of the electric field at the AlN core in the higher order bands, once the AlN dielectric functions present increasing values of $\delta \varepsilon / \delta \lambda$ at higher energies.

The gap-midgap ratio and the midgap frequency of both the ideal oxide-cladding AlN PhC presented here and of an air-bridge AlN PhC slab structure designed for comparative purposes are shown in Table II. The first order corrected gap-midgap ratios in the $\Gamma-K$ and $\Gamma-M$ directions of the proposed device are $21.09 \%$ and $25.59 \%$, respectively. These values are around 20\% lower than the ones of the optimum air-bridge AlN PhC slab structure. The values of the midgap frequencies in the $\Gamma-K$ and $\Gamma-M$ directions of the proposed structure are also lower than the air-bridge ones by roughly $7 \%$. In this way, we verify that the oxidecladding has the effect of lowering the gap-midgap ratio, as depicted in Fig. 3, and also the midgap frequency. These will result in the increase of the intrinsic losses in some amount and in feature sizes near $7 \%$ smaller in comparison with the ones of the air-bridge structure.

Thus, for a $450 \mathrm{~nm}$ operating wavelength at the midgap frequency $a / \lambda=0.4345$, the ideal oxide-cladding AlN PhC slab structure parameters are: $a=195.5 \mathrm{~nm}, r=68.4 \mathrm{~nm}$, $h=176.0 \mathrm{~nm}$, and $h_{c}=156.4 \mathrm{~nm}$.

Concerning fabrication induced disorder in the lithography process, a comparison was realized between the transmission, reflection, and radiation coefficients calculated at the center of the PBG in the $\Gamma-K$ direction with normalized standard deviations $0.02 \leq \sigma / a \leq 0.08$. Fig. 5 shows the

TABLE II. Comparison of the gap-midgap ratio and midgap frequency of both the proposed device and the air-bridge AlN PhC.

\begin{tabular}{lccccc}
\hline \hline & \multicolumn{2}{c}{$\Gamma-M$ direction } & & \multicolumn{2}{c}{$\Gamma-K$ direction } \\
\cline { 2 - 3 } \cline { 6 - 6 } Device & $\Delta \omega / \omega_{m}$ & $\omega_{m}$ & & $\Delta \omega / \omega_{m}$ & $\omega_{m}$ \\
\hline Oxide-cladding & $25.59 \%$ & 0.4020 & & $21.09 \%$ & 0.4345 \\
Air-bridge & $30.62 \%$ & 0.4336 & & $26.03 \%$ & 0.4646 \\
\hline \hline
\end{tabular}



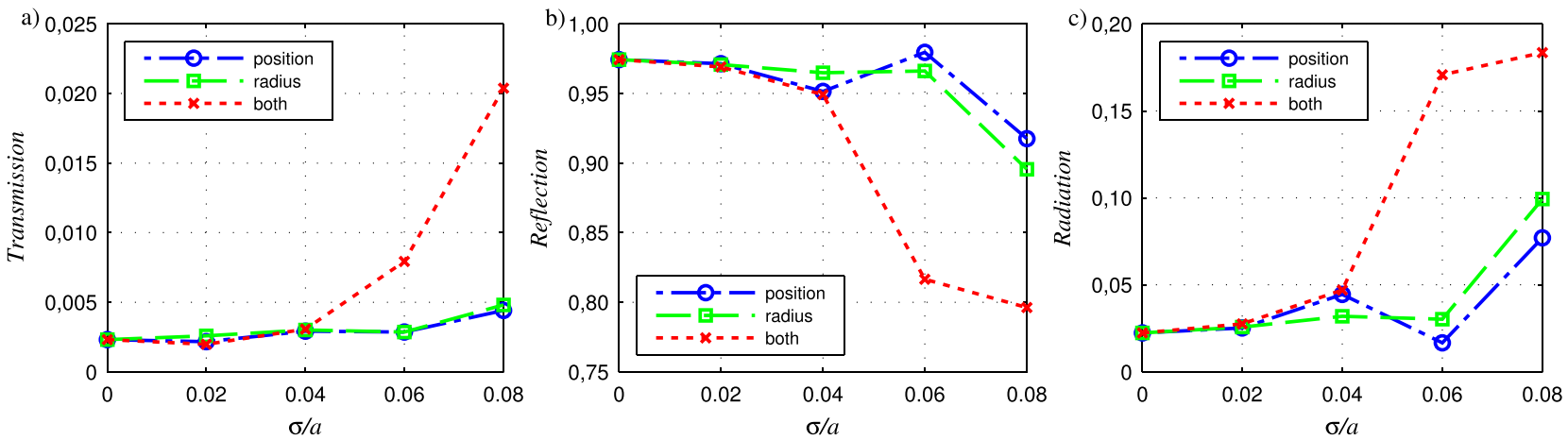

FIG. 5. Comparison of fluxes (a) transmitted, (b) reflected, and (c) radiated in a PhC slab structure subjected to fabrication induced disorder on position, radius, and both position and radius. The values were averaged around the center of the PBG in the $\Gamma-K$ direction.

values of transmission, reflection, and radiation coefficients for the designed $\mathrm{PhC}$ slab structure in which deviations were separately introduced in holes radii, positions, and both radii and positions. A threshold-like behavior in the amount of disorder was observed, as already reported in a high indexcontrast $\mathrm{PhC}$ using a uniform distribution to introduce displacements on sizes ${ }^{18}$ or positions ${ }^{19}$ of the holes separately. But here, it was also verified that a much more critical situation occurs when disorder is present simultaneously on positions and sizes of the holes. In this case, a lower threshold limit occurs, as can be seen in Fig. 5(c), in which for $\sigma / a$ $=0.06$ the radiation losses are 7 times as high as the ones observed when the disorder is introduced only in holes positions or radii. The increase in radiation loss produces a proportional reduction in reflection (Fig. 5(b)), whereas the transmission is almost unchanged (Fig. 5(a)).

The effects of disorder introduced in both positions and sizes of the holes, with standard deviations $\sigma_{p} / a=\sigma_{r} / a$ $=\sigma_{b} / a$ ranging from 0.02 to 0.08 , can be observed in detail in Fig. 6. We will conduct the analysis of these spectra in
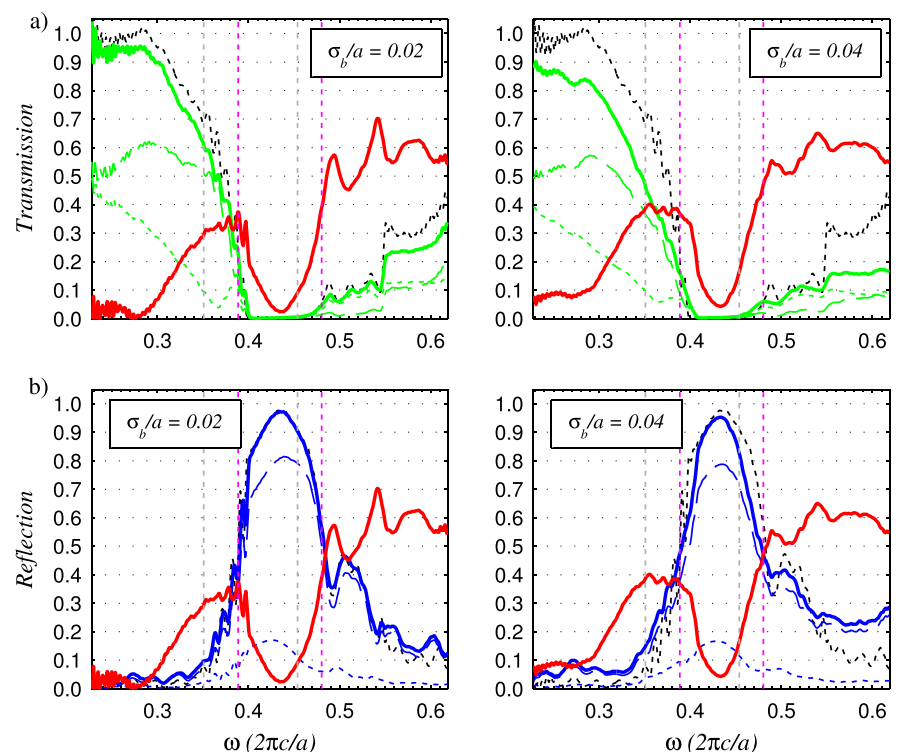

three distinct regions: the spectral range at the PBG and below and above it.

Below the PBG predominate the extended Bloch states, that in the ideal PhC structure have near $100 \%$ transmission. For little disorder $\left(\sigma_{b} / a=0.02\right)$ the total transmission and reflection curves are very close to the ideal ones, and it is possible to observe that the straight transmission dominates and it is almost constant, whereas the scattered states are continuously coupling into radiation states to the extent that frequency is approaching the lower edge of the PBG and so, the group velocity is becoming lower. As the disorder increases, a degradation in the straight transmission coefficient takes place and a linear raising in the reflection and radiation spectra appears as a consequence of the breaking in the discrete translational symmetry and no more conservation of the in-plane Bloch wavevectors.

At the center of the PBG in the $\Gamma-K$ direction, nearly $100 \%$ of the electromagnetic radiation flux is reflected in the ideal $\mathrm{PhC}$ structure. For small amounts of disorder $\left(0.02 \leq \sigma_{s} / a \leq 0.04\right)$, the reflection peaks become narrower,
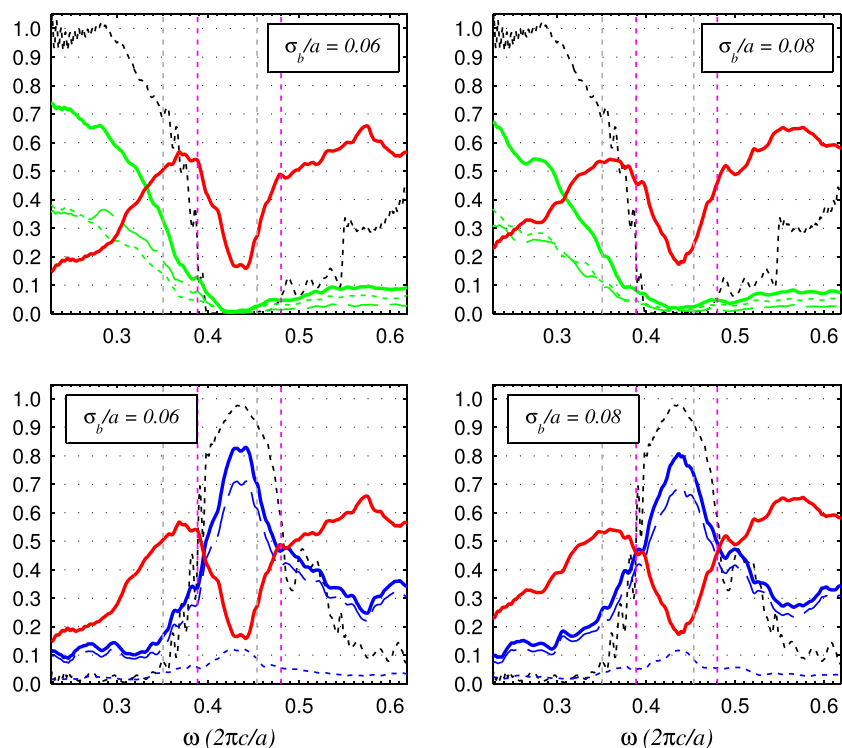

FIG. 6. Electromagnetic flux spectra of PhC slabs with disorder induced in both positions and sizes of the holes. The spectra were averaged over six random configurations of disorder simulated for each value of normalized standard deviation $\sigma_{b} / a$. (a) Spectra of total transmission (solid green), straight transmission (dashed green), and scattered transmission (dotted green). (b) Spectra of total reflection (solid blue), straight reflection (dashed blue), and scattered reflection (dotted blue). In all the figures, the radiation (solid red) and the ideal PhC (dotted black) spectra were inserted, besides the lower and upper bounds of the PBG in directions $\Gamma-K$ (dotted fuchsia line) and $\Gamma-M$ (dotted gray line). 

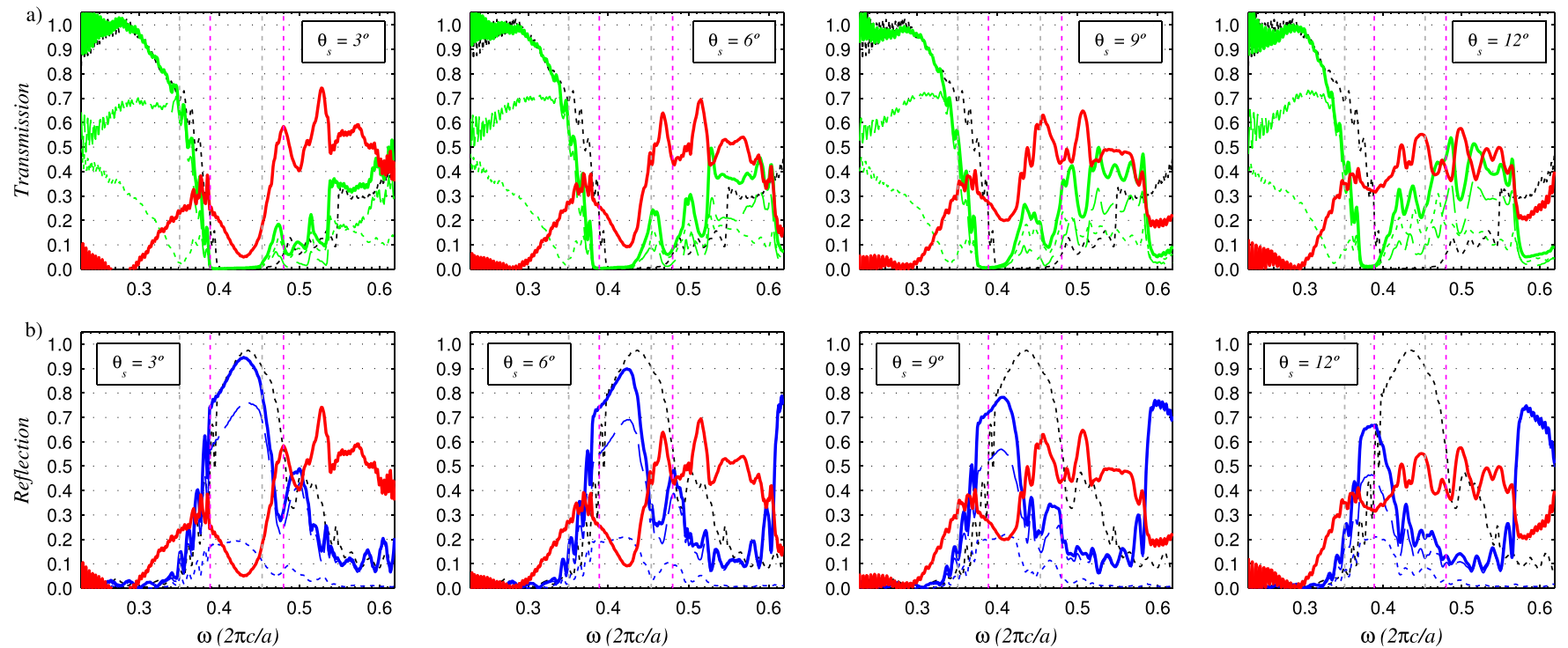

FIG. 7. Electromagnetic flux spectra of PhC slabs with holes sidewall sloped at $\theta_{s}$. (a) Spectra of total transmission (solid green), straight transmission (dashed green), and scattered transmission (dotted green). (b) Spectra of total reflection (solid blue), straight reflection (dashed blue), and scattered reflection (dotted blue). In all the figures, the radiation (solid red) and the ideal PhC (dotted black) spectra were inserted, besides the lower and upper bounds of the PBG in directions $\Gamma-K$ (dotted fuchsia line) and $\Gamma-M$ (dotted gray line).

but their amplitude at the PBG center is almost constant, as shown in Fig. 6(b). This occurs due to an increase of the local density of states around the upper and lower edges of the PBG, which makes the appearance of edge-states more likely. ${ }^{19}$ Higher levels of disorder allow the insertion of localized states at the center of the PBG and so, the reflection peaks become narrower, and there is also a great reduction in their amplitudes with a proportional increase in the radiation coefficients. The ratio between the straight and scattered reflection coefficients is almost unchanged with the increase in disorder level. Although modifying profoundly the shape of the reflection peaks, higher levels of disorder almost do not change their central frequency.

Above the upper edge of the PBG radiation losses dominate. The ideal $\mathrm{PhC}$ transmission curve increases in two steps corresponding to the light lines in the $\Gamma-M$ and $\Gamma-K$ directions. Above the light lines, there are radiation states that shrink as they propagate. The higher the disorder level, the larger the reflection coefficients leading to a decrease in the transmission of these radiation states.

We proceed now with the analysis of the effects produced by the sloped sidewalls in $\mathrm{PhC}$ holes resulting from dry etching processes. In Fig. 7 the spectra of the transmission and reflection coefficients for tilt angles $\left(\theta_{s}\right)$ ranging from $3^{\circ}$ to $12^{\circ}$ are exhibited. In the spectral range below the $\mathrm{PBG}$, there are no relevant modifications in the transmission and reflection profiles with increasing slope; they are closely related to the ideal $\mathrm{PhC}$ ones. No significant alterations occur to the ratio between the straight and scattered transmission also, with the former prevailing over the latter.

But the raising of the slope angle shifts the PBG to lower frequencies and also makes it narrower. This can be better observed in Fig. 7(b), through the reflection spectra. In the ideal $\mathrm{PhC}$, the maximum amplitude of the reflection coefficients is well centered at the PBG in the $\Gamma-K$ direction. The greater the $\theta_{s}$, the narrower the reflection peak and the higher its shift into lower frequencies. The amplitude of the reflection peaks also diminishes as a consequence of a linear increment in the radiation losses. Above the PBG, a higher order stop band arises in the continuum of radiation states following the shift of the PBG into lower frequencies.

The variational principle can explain very well the shift of the PBG into lower frequencies. As shown in Fig. 1(b), dielectric material is added in a region previously occupied by air when $\theta_{s}>0$, so that the electric field energy located inside the higher dielectric material will increase and push the eigenstates into lower frequencies. A similar effect is obtained by shrinking the sizes of the holes to fit the radius measured at the center of the AIN sloped sidewall. Using the PWE we obtained the midgap frequencies and the gapmidgap ratios from the band diagrams of $\mathrm{PhC}$ structures with reduced radius and $\theta_{s}=0$. These values are compared in Fig. 8 with the ones from the FDTD spectra. A good

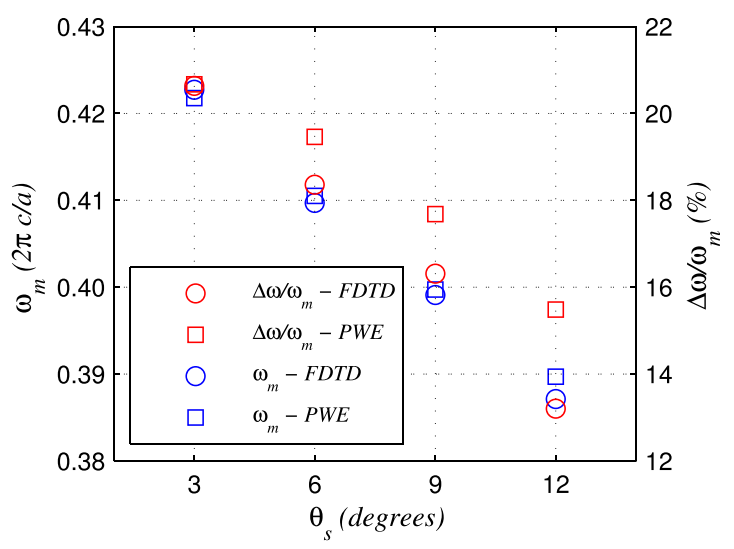

FIG. 8. Midgap frequencies and gap-midgap ratios calculated from FDTD simulations (circular marks) and through the band diagrams of $\mathrm{PhC}$ structures in which the holes radii was adjusted to fit the ones measured at the center of the AlN slab with sloped sidewalls (squared marks). 

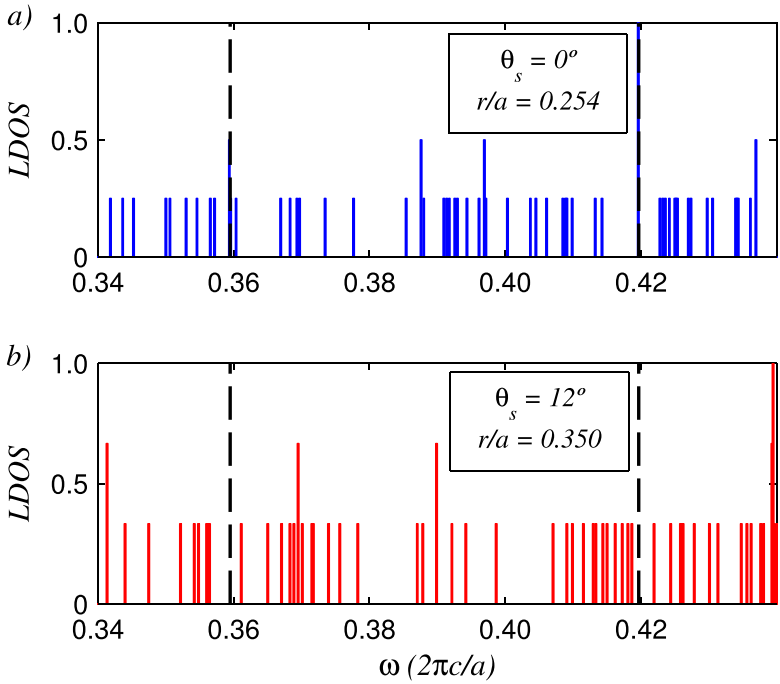

FIG. 9. Local density of states of (a) a $\mathrm{PhC}$ with reduced hole size without slope and (b) a $\mathrm{PhC}$ with ideal hole size and $\theta_{s}=12^{\circ}$.

agreement was verified between the midgap frequencies, but structures with sloped sidewalls have lower values of gapmidgap ratios.

In Fig. 9 it can be verified that there is a higher density of states around the edges of the PBG in the sloped sidewall $\mathrm{PhC}$ (Fig. 9(b)) than in a $\mathrm{PhC}$ with reduced radius and uniform holes (Fig. 9(a)). The holes with sloped sidewalls break the mirror symmetry about the central $x y$ plane, so that the probability of localized states around the edges of the PBG grows and yields the narrower PBG. This can be verified in Fig. 8 and also through the comparison of the solid blue and the dashed red curves in Fig. 10. The reduction in gapmidgap ratio leads to an increase in decay length of the evanescent modes inside the $\mathrm{PhC}$, so that they penetrate more deeply into the crystal body and couple into radiation states, explaining the accentuated growth of the radiation losses inside the PBG for the larger $\theta_{s}$ in Fig. 7.

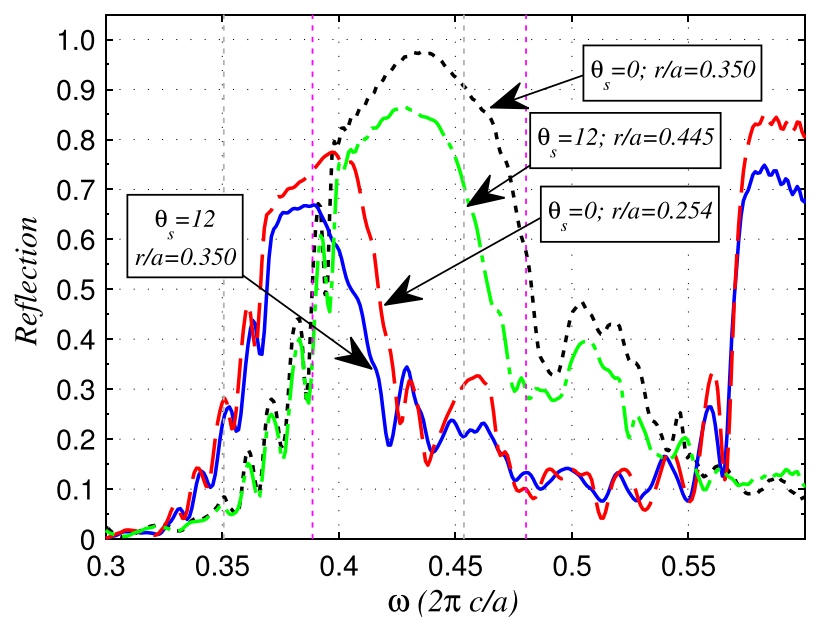

FIG. 10. Reflection spectra of ideal PhC structure $\left(\theta_{s}=0\right.$ and $r / a=0.350$ dotted black), sloped sidewall PhC $\left(\theta_{s}=12\right.$ and $r / a=0.350$-solid blue), reduced hole radius $\mathrm{PhC}$ without slope $\left(\theta_{s}=0\right.$ and $r / a=0.254$-dashed red), and increased radius $\mathrm{PhC}$ with sloped sidewalls $\left(\theta_{s}=12\right.$ and $r / a=0.445$-dashed dotted green).

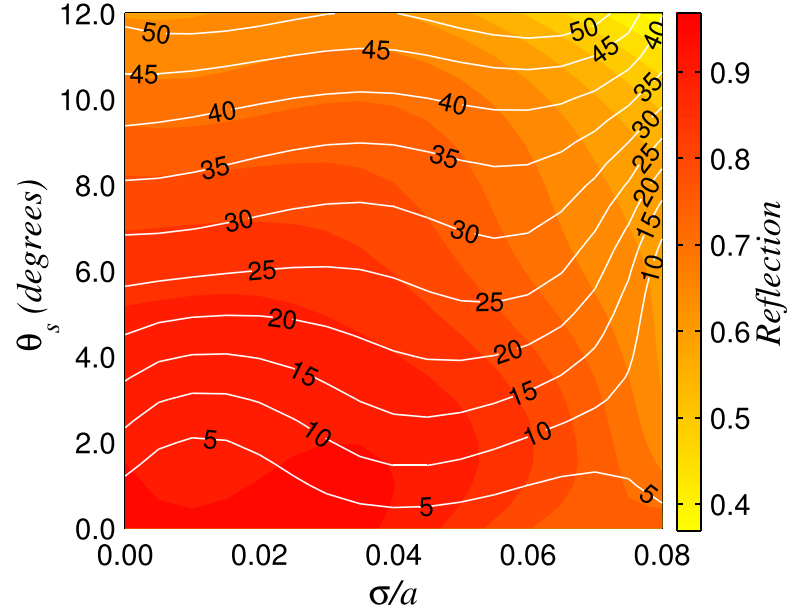

FIG. 11. Reflection coefficients (filled contours) and shifts of the midgap frequency with respect to the PBG size of the ideal $\mathrm{PhC}$ structure (white isolines) in PhC structures subjected to different values of $\sigma_{p}, \sigma_{r}$, and $\theta_{s}$.

A possible solution to mitigate the effects of slope in holes sidewall is increasing the holes radii in such a way that at the center of the $\mathrm{PhC}$ core they match the value of an ideal hole size. In Fig. 10, this can be verified by comparing the positions of the curve corresponding to an ideal $\mathrm{PhC}$ structure $\left(\theta_{s}=0\right.$ and $r / a=0.350$-dotted black curve) and that related to a $\mathrm{PhC}$ with $\theta_{s}=12^{\circ}$, and $r / a$ increased to 0.445 (dashed dotted green curve). Besides the fact that the midgap frequencies coincide very well, the latter still presents a narrower $\mathrm{PBG}$ due to the reasons already mentioned.

Comparing the data on Figs. 6 and 7, it is also possible to assert that the deviations in sizes and positions of the holes impacts more deeply the extended states than the slope in holes sidewalls, whereas the latter has a more significant influence on the evanescent states than the former.

Finally, in Fig. 11 it is possible to assess the response of $\mathrm{PhC}$ structures in which both the displacement in $\mathrm{PhC}$ holes sizes and positions are introduced as well as sloped sidewalls. This figure shows the amplitudes of the reflection peaks at the center of the PBG and also the shifts in the midgap frequencies of the disordered $\mathrm{PhC}$ with respect to the PBG size of the ideal PhC slab already designed. The threshold-like behavior for the disorder induced in lithography can be verified by the faster decrease of the reflection coefficients for $\sigma / a \geq 0.06$. But, even for PhC structures with $\sigma / a=0$, a similar degree of decrease in the reflection coefficients is present in $\mathrm{PhC}$ structures with $\theta_{s} \geq 9^{\circ}$. The reflection coefficients decay for values as low as 0.5 when the $\mathrm{PhC}$ structure experiences values of $\sigma / a \geq 0.06$ and $\theta_{s}$ $\geq 9^{\circ}$ at the same time. However, the impacts of slope in holes sidewalls are more critical than the displacements in holes sizes and positions. Indeed, this can be seen by the almost linear shifts in the midgap frequencies with the raising of the slope angles.

\section{CONCLUSIONS}

The dispersion functions of the AIN measured by VASE were used for adjusting the band diagram of the proposed 
oxide-cladding AlN PhC slab. Negligible shifts in the lower order bands were given by the first order perturbation theory correction. Thus, for operation at the midgap frequency, the AlN dispersion will not impact considerably the design. The first order corrected gap-midgap ratios in the $\Gamma-K$ and $\Gamma-M$ directions are $21.09 \%$ and $25.59 \%$, respectively. These values are around $20 \%$ lower than the ones of an optimum air-bridge AlN PhC slab structure.

We have also presented a detailed assessment of the effects of fabrication induced disorder in the spectral response of the proposed device. Fluctuations in holes sizes and positions were considered as well as slope in holes sidewalls. The lithography process is more critical for the extended states in the $\mathrm{PhC}$, whereas the dry etching process might impact the evanescent states more deeply, as it produces an increase in radiation losses and also a linear shift in the midgap frequency. However, we have verified that enlarging the holes sizes might mitigate these shifts in the midgap frequencies.

For the designed device, values of $\sigma_{p} / a$ and $\sigma_{r} / a$ as low as 0.05 can be tailored in the lithography process. This value represents a maximum standard deviation of near $10 \mathrm{~nm}$. Ideally, after the dry etching process the slope in the holes sidewalls should not exceed $5^{\circ}$. Inside these limits, the reflection coefficients should stay above 0.9 with low radiation loss and the shift in the midgap frequency should not be higher than $20 \%$.

\section{ACKNOWLEDGMENTS}

We would like to thank the Conselho Nacional de Desenvolvimento Científico e Tecnológico (CNPq) and Financiadora de Estudos e Projetos (FINEP) for the financial support.

${ }^{1}$ R. D. Meade, A. Devenyi, J. D. Joannopoulos, O. L. Alerhand, D. A. Smith, and K. Kash, J. Appl. Phys. 75, 4753 (1994).

${ }^{2}$ S. G. Johnson, S. Fan, P. R. Villeneuve, J. D. Joannopoulos, and L. A. Kolodziejski, Phys. Rev. B 60, 5751 (1999).

${ }^{3}$ K. McGroddy, A. David, E. Matioli, M. Iza, S. Nakamura, S. DenBaars, J. S. Speck, C. Weisbuch, and E. L. Hu, Appl. Phys. Lett. 93, 103502 (2008). ${ }^{4}$ N. Ganesh, I. D. Block, and B. T. Cunningham, Appl. Phys. Lett. 89, 023901 (2006).
${ }^{5}$ N. Ganesh, W. Zhang, P. C. Mathias, E. Chow, J. A. N. T. Soares, V. Malyarchuk, A. D. Smith, and B. T. Cunningham1, Nat. Nanotechnol. 2, 515 (2007).

${ }^{6}$ C. Xiong, W. H. P. Pernice, X. Sun, C. Schuck, K. Y. Fong, and H. X. Tang, New J. Phys. 14, 095014 (2012).

${ }^{7}$ H. Jung, C. Xiong, K. Y. Fong, X. Zhang, and H. X. Tang, Opt. Lett. 38, 2810 (2013)

${ }^{8}$ C. P. Ho, P. Pitchappa, B. W. Soon, and C. Lee, Opt. Express 23, 10598 (2015).

${ }^{9}$ N. Watanabe, T. Kimoto, and J. Suda, J. Appl. Phys. 104, 106101 (2008).

${ }^{10}$ S. Figge, H. Kröncke, D. Hommel, and B. M. Epelbaum, Appl. Phys. Lett. 94, 101915 (2009).

${ }^{11}$ A. Jacquot, B. Lenoir, A. Dauscher, P. Verardi, F. Craciun, M. Stölzer, M. Gartner, and M. Dinescu, Appl. Surf. Sci. 186, 507 (2002).

${ }^{12}$ D. Sam-Giao, D. Néel, S. Sergent, B. Gayral, M. J. Rashid, F. Semond, J. Y. Duboz, M. Mexis, T. Guillet, C. Brimont, S. David, X. Checoury, and P. Boucaud, Appl. Phys. Lett. 100, 191104 (2012).

${ }^{13}$ W. H. P. Pernice, C. Xiong, C. Schuck, and H. X. Tang, Appl. Phys. Lett. 100, 091105 (2012).

${ }^{14}$ D. Néel, I. Roland, X. Checoury, M. E. Kurdi, S. Sauvage, C. Brimont, T. Guillet, B. Gayral, F. Semond, and P. Boucaud, Adv. Nat. Sci. 5, 023001 (2014).

${ }^{15}$ S. G. Johnson, P. R. Villeneuve, S. Fan, and J. D. Joannopoulos, Phys. Rev. B 62, 8212 (2000).

${ }^{16}$ R. Ferrini, D. Leuenberger, R. Houdré, H. Benisty, M. Kamp, and A. Forchel, Opt. Lett. 31, 1426 (2006).

${ }^{17}$ W. Bogaerts, P. Bienstman, and R. Baets, Opt. Lett. 28, 689 (2003).

${ }^{18}$ M. A. Kaliteevski, J. M. Martinez, D. Cassagne, and J. P. Albert, Phys. Status Solidi A 195, 612 (2003).

${ }^{19}$ D. M. Beggs, M. A. Kaliteevski, R. A. Abram, D. Cassagne, and J. P. Albert, J. Phys.: Condens. Matter 17, 1781 (2005).

${ }^{20}$ S. Hughes, L. Ramunno, J. F. Young, and J. E. Sipe, Phys. Rev. Lett. 94, 033903 (2005).

${ }^{21}$ W. Fan, Z. Hao, Z. Li, Y. Zhao, and Y. Luo, J. Lightwave Technol. 28, 1455 (2010).

${ }^{22}$ M. V. Pelegrini, M. A. Alvarado, M. I. Alayo, and I. Pereyra, Can. J. Phys. 92, 940 (2014).

${ }^{23}$ S. Shokhovets, R. Goldhahn, G. Gobsch, S. Piekh, R. Lantier, A. Rizzi, V. Lebedev, and W. Richter, J. Appl. Phys. 94, 307 (2003).

${ }^{24}$ C. R. Aita, C. J. G. Kubiak, and F. Y. H. Shih, J. Appl. Phys. 66, 4360 (1989).

${ }^{25}$ W. Wu and B. Chiou, Semicond. Sci. Technol. 11, 1317 (1996).

${ }^{26}$ E. G. Melo and M. I. Alayo, Photonics Nanostruct. - Fundam. Appl. 14, 35 (2015).

${ }^{27}$ S. Johnson and J. Joannopoulos, Opt. Express 8, 173 (2001).

${ }^{28}$ T. Xu, M. S. Wheeler, H. E. Ruda, M. Mojahedi, and J. S. Aitchison, Opt. Express 17, 8343 (2009).

${ }^{29}$ S. G. Johnson, M. Ibanescu, M. A. Skorobogatiy, O. Weisberg, J. D. Joannopoulos, and Y. Fink, Phys. Rev. E 65, 066611 (2002).

${ }^{30}$ A. F. Oskooi, D. Roundy, M. Ibanescu, P. Bermel, J. D. Joannopoulos, and S. G. Johnson, Comput. Phys. Commun. 181, 687 (2010). 